\title{
The domination number of the graph defined by two levels of the $n$-cube
}

\author{
Leila Badakhshian* \\ Department of Mathematics, Tafresh University, \\ Tafresh, Iran \\ Gyula O.H. Katona ${ }^{\dagger}$ \\ MTA Rényi Institute, Budapest, Hungary \\ and \\ Zsolt Tuza \\ MTA Rényi Institute, Budapest, Hungary \\ and \\ Department of Computer Science and Systems Technology \\ University of Pannonia, Veszprém, Hungary
}

\begin{abstract}
Consider all $k$-element subsets and $\ell$-element subsets $(k>\ell)$ of an $n$-element set as vertices of a bipartite graph. Two vertices are adjacent if the corresponding $\ell$-element set is a subset of the corresponding $k$-element set. Let $G_{k, \ell}$ denote this graph The domination number of $G_{k, 1}$ will exactly be determined. We also give lower and upper estimates on the domination number $G_{k, 2}$ and pose a conjecture of asymptotic nature.
\end{abstract}

*This work is a part of $\mathrm{PhD}$ thesis of the first author at the Tafresh university under the direction of Prof.D.AMojdeh and Dr.M.Habibi.

${ }^{\dagger}$ The research of this author was supported by the National Research, Development and Innovation Office - NKFIH Fund No's SSN117879, NK104183 and K116769.

${ }^{\ddagger}$ Research supported in part by the National Research, Development and Innovation Office - NKFIH under the grant SNN 116095, and by the Széchenyi 2020 grant EFOP3.6.1-16-2016-00015. 


\section{Introduction}

Let $[n]=\{1,2, \ldots, n\}$ be our underlying set and let $\left(\begin{array}{c}{[n]} \\ k\end{array}\right)$ denote the family of all $k$-element subsets of $[n]$. Suppose $n>k>\ell \geq 1$. Consider the bipartite graph $G=\left(\left(\begin{array}{c}{[n]} \\ k\end{array}\right),\left(\begin{array}{c}{[n]} \\ \ell\end{array}\right) ; E\right)$ where the vertices $A \in\left(\begin{array}{c}{[n]} \\ k\end{array}\right)$ and $B \in\left(\begin{array}{c}{[n]} \\ \ell\end{array}\right)$ are adjacent iff $A \supset B$. This graph will be denoted by $G_{k, \ell}$. The family $\left(\begin{array}{c}{[n]} \\ k\end{array}\right)$ is often called the $k$ th level of the $n$-cube. Then it is not much misleading to call $G_{k, \ell}$ as the graph defined by the $k$ th and $\ell$ th level.

We say that a vertex $v$ dominates the vertex $u$ in a graph $G(V, E)$ if either $u=v$ or $\{u, v\} \in E$. A subset $D$ of $V$ is dominating the graph if every vertex $u \in V$ is dominated by at least one element $v$ of $D$. The domination number $\gamma(G)$ of a graph $G$ is the smallest possible size of a dominating set. The goal of the present paper is to study $\gamma\left(G_{k, \ell}\right)$ for some small values of $k$ and $\ell$.

In Section 2 we will determine the exact value of $\gamma\left(G_{k, 1}\right)$.

Theorem $1 \gamma\left(G_{k, 1}\right)=n-k+1$ holds for $k \geq 2$.

It seems to be much more difficult to determine $\gamma\left(G_{k, 2}\right)$. We have the following conjecture of asymptotical nature.

Conjecture $1 \gamma\left(G_{k, 2}\right)=\frac{k+3}{2(k-1)(k+1)} n^{2}+o\left(n^{2}\right)$ holds for $k \geq 3$.

Unfortunately we are able to prove only a slightly weaker lower bound for general $k$.

Theorem $2 \gamma\left(G_{k, 2}\right) \geq \frac{k^{2}+k-4}{2(k-1)^{2} k} n^{2}+o\left(n^{2}\right)$ holds for $k \geq 4$.

This theorem will be proved in Section 3.

Remark 1 Observe that the difference of the coefficients in Conjecture 1 and Theorem 2 is really tiny:

$$
\frac{2}{(k-1)^{2} k(k+1)}
$$

which tends to 0 by $k$ very fast.

For $k=3$ the constants in Conjecture 1 and Theorem 2 are $\frac{3}{8}$ and $\frac{1}{3}$, respectively. We can slightly improve the latter one, see Section 4. 
Theorem $3 \gamma\left(G_{3,2}\right) \geq \frac{7}{20} n^{2}+o\left(n^{2}\right)$.

Section 5 contains some upper estimates on $\gamma\left(G_{k, 2}\right)$.

Theorem $4 \gamma\left(G_{3,2}\right) \leq \frac{3}{8} n^{2}-\frac{3}{4} n-\frac{1}{8}$.

Theorem $5 \gamma\left(G_{4,2}\right) \leq \frac{7}{30} n^{2}+o\left(n^{2}\right)$.

These two constructions meet the respective constants in Conjecture 1, however for general $k$, unfortunately, we have only a weak construction.

Theorem $6 \gamma\left(G_{k, 2}\right) \leq \frac{k+1}{2(k-1)^{2}} n^{2}+o\left(n^{2}\right)$ holds for $k \geq 3$.

\section{The easy case: $\ell=1$.}

Proof of Theorem 1. Let us start with a construction. Choose one $k$ element set $K$ and all elements of $[n]-K$ and consider them as vertices of $G_{k, 1}$. It is easy to see that this is a dominating set in this graph, therefore we have

$$
\gamma\left(G_{k, 1}\right) \leq n-k+1
$$

In order to prove the opposite direction, we have to verify that every dominating set in $G_{k, 1}$ has at least $n-k+1$ vertices. Suppose that a family $\mathcal{K}$ of $k$-element sets and the set $R \subset[n]$ form a dominating set in $G_{k, 1}$. We have to prove

$$
|\mathcal{K}|+|R| \geq n-k+1 .
$$

The pair $(\mathcal{K}, R)$ is a dominating set if and only if the following two conditions hold.

$$
\text { If } L \in\left(\begin{array}{c}
{[n]} \\
k
\end{array}\right), L \notin \mathcal{K} \text { then } L \cap R \neq \emptyset .
$$

$$
\text { If } i \in[n]-R \text { then there is a } K \in \mathcal{K} \text { such that } i \in K \text {. }
$$

If $|R| \geq n-k+1$ we are done. Therefore we can suppose that $|R|=n-k-t$ where $t \geq 0$. By (i) every set $L \in\left(\begin{array}{c}{[n]-R} \\ k\end{array}\right)$ must be in $\mathcal{K}$. Hence we have $|\mathcal{K}| \geq\left(\begin{array}{c}k+t \\ k\end{array}\right)=\left(\begin{array}{c}k+t \\ t\end{array}\right) \geq\left(\begin{array}{c}1+t \\ t\end{array}\right)=t+1$ and $|\mathcal{K}|+|R| \geq t+1+n-k-t=n-k+1$ as desired in (1). 


\section{Asymptotic lower bound on $\gamma\left(G_{k, 2}\right)$}

The proof will be based on a stronger version of the Turán theorem. For sake of completeness let us start with formulating the original theorem of Turán. Let $T(n, s)$ denote the following graph. Partition the set $[n]$ into $s$ almost equal (differences of the sizes are at most one) parts: $V=V_{1} \cup V_{2} \cup \ldots \cup V_{s}$. Two vertices are adjacent if and only if they are in distinct $V_{i}$ 's. The number of edges of $T(n, s)$ is denoted by $t(n, s)$.

Theorem 7 [7] If the graph with $n$ vertices contains no complete graph $K_{k}$ as a subgraph then the number of edges cannot exceed $t(n, k-1)$ and one can have equality only for $T(n, k-1)$.

If one more edge is added to $T(n, k-1)$ then it creates asymptotically $\left(\frac{n}{k-1}\right)^{k-2}$ copies of $K_{k}$. It is natural to guess that if $m$ new edges are added then $m\left(\frac{n}{k-1}\right)^{k-2}$ copies of $K_{k}$ are obtained, if $m$ is not too large. The quantity $t(n, k-1)$ is asymptotically equal to $\left(\begin{array}{l}n \\ 2\end{array}\right)\left(1-\frac{1}{k-1}\right)$. Let $m$ also be given in an asymptotic form $m=\mathrm{cn}$. Our above guess can be formulated in the following statement that can be easily obtained from Theorem 4 (see also Theorem 1) of a paper of Lovász and Simonovits.

Corollary 1 ( of a theorem of Lovász, Simonovits, [5]). If the graph $G=$ $(V, E)$ has $n$ vertices and at least

$$
|E|=\left(\begin{array}{l}
n \\
2
\end{array}\right)\left(1-\frac{1}{k-1}\right)+c n
$$

where $c<\frac{1}{k-1}$ then $G$ contains at least

$$
c \frac{n^{k-1}}{(k-1)^{k-2}}+o\left(n^{k-1}\right)
$$

copies of $K_{k}$.

This corollary will actually be used for the complementing graph, as follows.

Corollary 2 If the graph $G=(V, E)$ has $n$ vertices and at most

$$
|E|=\left(\begin{array}{l}
n \\
2
\end{array}\right) \frac{1}{k-1}-c n
$$


where $c<\frac{1}{k-1}$ then $G$ contains at least

$$
c \frac{n^{k-1}}{(k-1)^{k-2}}+o\left(n^{k-1}\right)
$$

"empty" copies of $K_{k}$.

Proof of Theorem 2. Consider a pair $(\mathcal{K}, E)$ where $\mathcal{K}$ is a family of $k$-element subsets of $[n]$ and $E \subset[n]$ is a set of pairs $\{i, j\}(i, j \in[n]$ ) (edges of the graph $G=([n], E))$. Such a pair $(\mathcal{K}, E)$ is a dominating set if and only if the following two conditions hold.

$$
\text { If } L \in\left(\begin{array}{c}
{[n]} \\
k
\end{array}\right), L \notin \mathcal{K} \text { then } L \text { includes an element of } E \text {. }
$$

If $\{i, j\} \notin E$ then there is a $K \in \mathcal{K}$ such that $\{i, j\} \subset K$.

We need to prove

$$
|\mathcal{K}|+|E| \geq \frac{k^{2}+k-4}{2(k-1)^{2} k} n^{2}+o\left(n^{2}\right)
$$

If $|\mathcal{K}| \geq \frac{k^{2}+k-4}{2(k-1)^{2} k} n^{2}$, we are done. Thus

$$
|\mathcal{K}| \leq \frac{k^{2}+k-4}{2(k-1)^{2} k} n^{2}
$$

can be supposed. Almost all $k$-element sets $L$ satisfy (iii), the edges in $E$ must "clinch" them with the exception of the members of $\mathcal{K}$, therefore the number of empty $k$-element sets in the graph defined by $E$ is at most $\frac{k^{2}+k-4}{2(k-1)^{2} k} n^{2}$ by (3). Here the inequality

$$
\frac{k^{2}+k-4}{2(k-1)^{2} k} n^{2}<\frac{k^{2}+k-4}{2(k-1)^{2} k} \cdot \frac{n^{k-1}}{(k-1)^{k-2}}
$$

holds for $k \geq 4$ if $n$ is large enough. Hence we know that there are less than

$$
\frac{k^{2}+k-4}{2(k-1)^{2} k} \cdot \frac{n^{k-1}}{(k-1)^{k-2}}
$$


empty $K_{k}$ in the graph $G$. Corollary 2 can be applied with

$$
c=\frac{k^{2}+k-4}{2(k-1)^{2} k}<\frac{1}{k-1} .
$$

The number of edges of $G$ must be more than $\left(\begin{array}{l}n \\ 2\end{array}\right) \frac{1}{k-1}-c n$ that is

$$
|E|>\left(\begin{array}{l}
n \\
2
\end{array}\right) \frac{1}{k-1}-\frac{k^{2}+k-4}{2(k-1)^{2} k} n .
$$

By (iv) every pair $\{i, j\}(1 \leq i, j \leq n)$ is either an edge of $G$ or is covered by a member of $\mathcal{K}$. One member can cover $\left(\begin{array}{l}k \\ 2\end{array}\right)$ pairs. Hence we have

$$
\left(\begin{array}{l}
k \\
2
\end{array}\right)|\mathcal{K}|+|E| \geq\left(\begin{array}{l}
n \\
2
\end{array}\right) \text {. }
$$

In other words

$$
|\mathcal{K}|+\frac{|E|}{\left(\begin{array}{l}
k \\
2
\end{array}\right)} \geq \frac{\left(\begin{array}{l}
n \\
2
\end{array}\right)}{\left(\begin{array}{l}
k \\
2
\end{array}\right)} .
$$

Add the suitable multiple of (5) to (6):

$$
\begin{gathered}
|\mathcal{K}|+|E|=|\mathcal{K}|+\frac{|E|}{\left(\begin{array}{c}
k \\
2
\end{array}\right)}+|E| \frac{\left(\begin{array}{l}
k \\
2
\end{array}\right)-1}{\left(\begin{array}{l}
k \\
2
\end{array}\right)} \geq \\
\left(\begin{array}{l}
n \\
2
\end{array}\right)\left(\frac{1}{\left(\begin{array}{c}
k \\
2
\end{array}\right)}+\frac{\left(\begin{array}{l}
k \\
2
\end{array}\right)-1}{\left(\begin{array}{l}
k \\
2
\end{array}\right)(k-1)}\right)+o\left(n^{2}\right)=\left(\begin{array}{l}
n \\
2
\end{array}\right) \frac{k^{2}+k-4}{2(k-1)^{2} k}+o\left(n^{2}\right),
\end{gathered}
$$

proving (2) and the theorem.

\section{Asymptotic lower bound on $\gamma\left(G_{3,2}\right)$}

The ideas of the previous proof will be followed, but we have to make some refinements at two places and have to use a celebrated theorem of Ruzsa and Szemerédi.

Theorem 8 [6] Let $\mathcal{H} \subset\left(\begin{array}{c}{[n]} \\ 3\end{array}\right)$ and suppose that it does not contain a threemember subfamily spanned by 6 elements of $[n]$. Then $|\mathcal{H}|=o\left(n^{2}\right)$. 
Proof of Theorem 3. We need to prove

$$
|\mathcal{H}|+|E| \geq \frac{7}{20} n^{2}+o\left(n^{2}\right)
$$

where $\mathcal{H} \subset\left(\begin{array}{c}{[n]} \\ 3\end{array}\right)$ and $E \subset\left(\begin{array}{c}{[n]} \\ 2\end{array}\right)$ satisfy the following conditions.

$$
\text { If } L \in\left(\begin{array}{c}
{[n]} \\
3
\end{array}\right), L \notin \mathcal{H} \text { then } L \text { includes an element of } E \text {. }
$$

If $\{i, j\} \notin E$ then there is an $H \in \mathcal{H}$ such that $\{i, j\} \subset H$.

Observe first that (4) does not hold here since $k-1=2$ in this case. Therefore we have to find another way to prove a statement similar to (5). Two cases will be distinguished.

If $|\mathcal{H}| \leq\left(\frac{1}{4}-\varepsilon\right) n^{2}(\varepsilon>0)$ then Corollary 2 can be used with $c=\frac{1}{2}-2 \varepsilon$, as before:

$$
|E|>\left(\begin{array}{l}
n \\
2
\end{array}\right) \frac{1}{2}-\left(\frac{1}{2}-2 \varepsilon\right) n .
$$

Suppose now

$$
|\mathcal{H}|>\left(\frac{1}{4}-\varepsilon\right) n^{2}(\varepsilon>0) .
$$

In this case we cannot prove a good lower estimate on $|E|$, like (8), but we can directly prove (7). Condition (v) implies

$$
(n-2)|E|+|\mathcal{H}| \geq\left(\begin{array}{l}
n \\
3
\end{array}\right)
$$

since every 3 -element set is either in $\mathcal{H}$ or can be obtained from an element of $E$ adding one element of $[n]-E$.

This and $|\mathcal{H}|<\frac{7}{20} n^{2}+o\left(n^{2}\right)$ result in

$$
|E| \geq \frac{n^{2}}{6}+o\left(n^{2}\right)
$$

(10) and (9) lead to $|E|+|\mathcal{H}| \geq\left(\frac{1}{6}+\frac{1}{4}-\varepsilon\right) n^{2}+o\left(n^{2}\right)$ which is stronger than (7). Inequality (8) can really be supposed.

We know $3|\mathcal{H}|+|E| \geq\left(\begin{array}{l}n \\ 2\end{array}\right)$ from (vi), but we will use an improved version. $\mathcal{H}$ will be partitioned into 3 parts. If $A, B \in \mathcal{H}$ satisfy $|A \cap B|=2$ then we call them two-intersecting. Let $\mathcal{H}_{2}$ consist of those members of $\mathcal{H}$ which are 
two-intersecting with another member. Define $\mathcal{H}_{1}$ as the set of members of $\mathcal{H}$ which are not two-intersecting with any other member, but one of its two element subsets is in $E$. Finally let $\mathcal{H}_{0}$ be the family of all other members of $\mathcal{H}$, that is the members which are not two-intersecting with any other member, and contain no element of $E$ as a subset.

$$
\mathcal{H}=\mathcal{H}_{0} \cup \mathcal{H}_{1} \cup \mathcal{H}_{2}
$$

is obvious. Now we will show some properties of these subfamilies. Define the shadow of $\mathcal{H}_{2}$ as $\sigma\left(\mathcal{H}_{2}\right)=\left\{\{i, j\}: i \neq j,\{i, j\} \subset H\right.$ for some $\left.H \in \mathcal{H}_{2}\right\}$.

\section{Lemma 1}

$$
\left|\sigma\left(\mathcal{H}_{2}\right)\right| \leq \frac{5}{2}\left|\mathcal{H}_{2}\right|
$$

Proof. We will count the number of pairs $(H, f)$ where $H \in \mathcal{H}_{2}, f \in$ $\sigma\left(\mathcal{H}_{2}\right), f \subset H$, in two different ways. Let $d(f)$ denote the number of members $H$ of $\mathcal{H}_{2}$ satisfying $f \subset H$.

$$
3\left|\mathcal{H}_{2}\right|=\sum_{f \in \sigma\left(\mathcal{H}_{2}\right)} d(f)
$$

Denote the number of $f^{\prime}$ 's $\left(f \in \sigma\left(\mathcal{H}_{2}\right)\right)$ with $d(f)=1$, that is the number of elements of $\sigma\left(\mathcal{H}_{2}\right)$ which are contained in exactly one member $H \in \mathcal{H}$, by $s$. Each $H$ has one two-element subset $f$ which is covered by another one too, that is $d(f) \geq 2$ holds. Therefore each $H$ can create at most two $f$ 's with $d(f)=1$ :

$$
s \leq 2\left|\mathcal{H}_{2}\right| .
$$

Then the right hand side of (13) can be lower-bounded in the following way:

$$
\sum_{f \in \sigma\left(\mathcal{H}_{2}\right)} d(f) \geq s+2\left(\left|\sigma\left(\mathcal{H}_{2}\right)\right|-s\right)=2\left|\sigma\left(\mathcal{H}_{2}\right)\right|-s .
$$

Combining (13), (14) and (15) we obtain

$$
2\left|\sigma\left(\mathcal{H}_{2}\right)\right|-3\left|\mathcal{H}_{2}\right| \leq 2\left|\mathcal{H}_{2}\right|,
$$

proving the statement.

Lemma $2\left|\mathcal{H}_{0}\right|=o\left(n^{2}\right)$. 
Proof. Theorem 8 will be used. Choose 6 elements of $[n]$, say the set $\{1,2,3,4,5,6\}$ and the members of $\mathcal{H}_{0}$ spanned by them. If two of them are vertex-disjoint then there is no third one, since the members of $\mathcal{H}_{0}$ do not share two-element sets. Therefore any two of them have one element in common. If $\{1,2,3,4,5,6\}$ spans 3 members then their system must be isomorphic with $\{\{1,2,3\},\{2,4,5\},\{3,5,6\}\}$. Here $\{2,3,5\} \notin \mathcal{H}_{0}$ consequently, by $(v)$ one of its two-element subsets, say $\{2,3\}$ is in $E$. However this is a subset of $\{1,2,3\}$. This contradiction shows that the conditions of Theorem 8 are satisfied.

Now, it is easy to finish the proof of the theorem using (vi). The members of $\mathcal{H}_{2}$ cover exactly $\left|\sigma\left(\mathcal{H}_{2}\right)\right|$ two-element subsets. A member of $\mathcal{H}_{1}$ covers at most two from the set $\left(\begin{array}{c}{[n]} \\ 2\end{array}\right)-E$. Finally a member of $\mathcal{H}_{0}$ can cover at most 3 two-element subsets. ( $v$ ) gives

$$
3\left|\mathcal{H}_{0}\right|+2\left|\mathcal{H}_{1}\right|+\left|\sigma\left(\mathcal{H}_{2}\right)\right|+|E| \geq\left(\begin{array}{l}
n \\
2
\end{array}\right) .
$$

By Lemma 1 we obtain

$$
\frac{5}{2}\left|\mathcal{H}_{0}\right|+2\left|\mathcal{H}_{1}\right|+\frac{5}{2}\left|\mathcal{H}_{2}\right|+|E| \geq\left(\begin{array}{l}
n \\
2
\end{array}\right)-\frac{1}{2}\left|\mathcal{H}_{0}\right| .
$$

(11) and Lemma 2 lead to $\frac{5}{2}|\mathcal{H}|+|E| \geq\left(\begin{array}{l}n \\ 2\end{array}\right)-o\left(n^{2}\right)$ that is

$$
|\mathcal{H}|+\frac{2}{5}|E| \geq \frac{2}{5}\left(\begin{array}{l}
n \\
2
\end{array}\right)-o\left(n^{2}\right)
$$

Add $\frac{3}{5}$ times (8) to obtain the statement of the theorem.

\section{Upper estimates: constructions}

Proof of Theorem 4. We will show the construction only for the case when $n$ is divisible by 4 . The dominating set will consist of a set $E$ of twoelement subsets and a family $\mathcal{H}$ of three-element subsets satisfying $(v)$ and (vi). Take a partition $X_{1} \cup X_{2}=[n]$ of equal sizes. Choose a perfect matching $M=\left\{e_{1}, e_{2}, \ldots, e_{\frac{n}{4}}\right\}$ in the complete graph defined by $X_{1}$. Define $E$ as the set of all pairs within one part, that is, it is the union of two complete graphs of sizes $\frac{n}{2}$ minus $M$. Let $\mathcal{H}$ consist of all three-element sets of the form $e_{i} \cup\{y\}$ where $1 \leq i \leq \frac{n}{4}, y \in X_{2}$. It is easy to see that this pair $(\mathcal{H}, E)$ 
satisfies conditions $(v)$ and $(v i)$, therefore they form a dominating set. On the other hand the total number of elements is

$$
2\left(\begin{array}{c}
\frac{n}{2} \\
2
\end{array}\right)-\frac{n}{4}+\frac{n}{4} \cdot \frac{n}{2}=\frac{3 n^{2}-6 n}{8} .
$$

The same construction gives a somewhat different result for $n$ 's not divisible by 4 . If the remainder is, $1,2,3$, then $\frac{3}{8}, 0$ and $-\frac{1}{8}$, respectively are added to the formula above.

Conjecture 2 We believe that these constructions give the exact values of $\gamma\left(G_{3,2}\right)$.

In order to give the construction for the general case we need a new concept and a related result from the literature. Consider a partition $X=$ $X_{1} \cup X_{2} \cup \ldots \cup X_{s}$ of our underlying set where $\left|X_{i}\right|=r$ holds for all $1 \leq i \leq s$. A family $\mathcal{T}(s, r)$ of subsets of $X$ is called a transversal design if

$$
\left|T \cap X_{i}\right|=1 \text { holds for all } T \in \mathcal{T}(s, r) \text { and } 1 \leq i \leq s
$$

and

for every pair $u \in X_{i}, v \in X_{j}(i \neq j)$ there is a unique member $T \in \mathcal{T}(s, r)$ such that $u, v \in T$.

It is easy to see that $|\mathcal{T}(s, r)|=r^{2}$.

Theorem 9 (see Hanani [4] ) If $r$ is a prime power, $s$ is a positive integer then there is a transversal design $\mathcal{T}(s, r)$.

Proof of Theorem 6. Suppose that $n$ is divisible by $k-1$ and $\frac{n}{k-1}$ is a prime power. Consider the partition $[n]=X_{1} \cup X_{2} \cup \ldots \cup X_{k-1}$. Then let $E$ consist of all pairs within one $X_{i}$ (that is $E$ is a union of $k-1$ vertex-disjoint complete graphs of size $\left.\frac{n}{k-1}\right)$. Define $\mathcal{K}$ in the following way: add an arbitrary element $a \notin T$ of $[n]$ to each $T \in \mathcal{T}\left(k-1, \frac{n}{k-1}\right)$.

Now condition (iii) obviously holds. By the definition of the transversal design (iv) also holds if $\mathcal{T}\left(k-1, \frac{n}{k-1}\right)$ is taken instead of $\mathcal{K}$. But the members of $\mathcal{T}\left(k-1, \frac{n}{k-1}\right)$ have $k-1$ elements, this is why we added an element to each member of $\mathcal{T}\left(k-1, \frac{n}{k-1}\right)$ to obtain $\mathcal{K}$.

Here $|E|=\frac{1}{2(k-1)} n^{2}+o\left(n^{2}\right)$ and $|\mathcal{K}|=\left(\frac{n}{k-1}\right)^{2}$ and these imply the statement of the theorem for the $n$ 's satisfying the conditions above.

In order to prove the statement for other $n$ 's the following theorem will be used. 
Theorem 10 [1] If $n>n_{0}$ then there is a prime number between $n$ and $n^{0.525}$.

Let $p(n)$ denote the largest prime number $\leq \frac{n}{k-1}$. The theorem above implies that

$$
(1-\varepsilon) \frac{n}{k-1} \leq p(n) \leq \frac{n}{k-1}
$$

holds for every $\varepsilon>0$ if $n$ is large enough. (Of course this latter statement is much weaker than Theorem 10 and can be deduced from the prime number theorem, but we wanted to show the strongest existing result in this direction.) We actually need the following consequence of (16).

$$
0 \leq n-p(n)(k-1) \leq \varepsilon n .
$$

The construction for $[n]$ is based on the construction for $[p(n)(k-1)]$. Let $\mathcal{K}$ and $E$ be the family of $k$-element subsets and pairs, respectively, constructed for $[p(n)(k-1)]$ in the first part of the proof. They satisfy (iii) and (iv) on $[p(n)(k-1)]$ and their total number is

$$
\frac{k+1}{2(k-1)^{2}} p^{2}(n)(k-1)^{2}+o\left(p^{2}(n)\right) \text {. }
$$

Add all the pairs containing at least one element from $[p(n)(k-1)+1, \ldots, n]$. This set of pairs is denoted by $E_{1}$. It is easy to see that $\mathcal{K}$ and $E \cup E_{1}$ satisfy both conditions (iii) and (iv) on $[n]$. Using (17) and (18) we obtain

$$
\begin{gathered}
|\mathcal{K}|+|E|+\left|E_{1}\right|=\frac{k+1}{2(k-1)^{2}} p^{2}(n)(k-1)^{2}+o\left(p^{2}(n)\right)+ \\
p(n)(k-1)(n-p(n)(k-1))+\left(\begin{array}{c}
n-p(n)(k-1) \\
2
\end{array}\right) \leq \\
\frac{k+1}{2(k-1)^{2}} n^{2}+o\left(n^{2}\right)+p(n)(k-1) \varepsilon n+\frac{1}{2}(\varepsilon n)^{2} \leq \\
\frac{k+1}{2(k-1)^{2}} n^{2}+o\left(n^{2}\right)+\left(\varepsilon+\frac{1}{2} \varepsilon^{2}\right) n^{2} .
\end{gathered}
$$

Since this inequality is valid for all $\varepsilon>0$, the desired inequality is obtained, the theorem is proved for all $n$.

Now we show our plan, how find constructions meeting the constants in Conjecture 1. Consider a partition $X=X_{1} \cup X_{2} \cup \ldots \cup X_{s}$ of our underlying 
set where $\left|X_{i}\right|=r$ holds for all $1 \leq i \leq s$. A family $\mathcal{T}^{+1}(s, r)$ of subsets of $X$ is called a +1 -transversal design if

$$
\left|T \cap X_{\ell}\right|=2 \text { but }\left|T \cap X_{i}\right|=1 \text { holds for all } T \in \mathcal{T}^{+1}(s, r) \text { and } i \in[s], i \neq \ell
$$

and

for every pair $u \in X_{i}, v \in X_{j}(i \neq j)$ there is a unique member $T \in \mathcal{T}^{+1}(s, r)$

$$
\text { such that } u, v \in T \text {. }
$$

Unlike in the case of a transversal design, where $|T|=s$ holds for the members of $\mathcal{T}$, here their sizes are $|T|=s+1$ in $\mathcal{T}^{+1}(s, r)$.

Now we show how to "blow up" a +1 -transversal design, that is having one, how to make bigger ones from it. Let $\mathcal{T}^{+1}(s, r)$ a +1 -transversal design on the partition $X_{i}(1 \leq i \leq s)$. Suppose that $q$ is a prime power and we will create a larger +1 -transversal design $\mathcal{T}^{+1}(s, q r)$ on the partition $Z_{1} \cup Z_{2} \cup$ $\ldots \cup Z_{s}$ where $Z_{i}$ is divided into parts $Z_{i}=X_{i}^{1} \cup X_{i}^{2} \cup \ldots \cup X_{i}^{q}$.

Suppose that $\mathcal{T}(s, q)$ is a transversal design on the partition $Y_{1} \cup Y_{2} \cup$ $\ldots \cup Y_{s}$ where $Y_{i}=\left\{y_{i, 1}, y_{i, 2}, \ldots y_{i, q}\right\}$. Define the combination $\mathcal{T}^{+1}(s, r) \Rightarrow$ $\mathcal{T}(s, q)$ of $\mathcal{T}^{+1}(s, r)$ and $\mathcal{T}(s, q)$ in the following way. Suppose that $T=$ $\left\{y_{1, j_{1}}, y_{2, j_{2}}, \ldots, y_{s, j_{s}}\right\} \in \mathcal{T}(s, q)$. Define $\mathcal{S}(T)$ as the family isomorphic to $\mathcal{T}^{+1}(s, r)$ on the partition $X_{1}^{j_{1}} \cup X_{2}^{j_{2}} \cup \ldots \cup X_{s}^{j_{s}}$. Then

$$
\mathcal{T}^{+1}(s, r) \Rightarrow \mathcal{T}(s, q)=\{\mathcal{S}(T): T \in \mathcal{T}(s, q)\}
$$

Lemma $3 \mathcal{T}^{+1}(s, r) \Rightarrow \mathcal{T}(s, q)$ is a +1 -transversal design on the partition $Z_{1} \cup Z_{2} \cup \ldots Z_{s}$.

Proposition 1 If there is an $r$ such that $\mathcal{T}^{+1}(k-1, r)$ exists then

$$
\gamma\left(G_{k, 2}\right) \leq \frac{k+3}{2(k-1)(k+1)} n^{2}+o\left(n^{2}\right) \text { holds for } k \geq 4
$$

meeting the constant of Conjecture 1. 
Proof. By Lemma 3 there is a +1 -transversal $\mathcal{T}^{+1}(k-1, q r)$ for every prime power $q$. Choose $n$ to be $(k-1) q r$. Let $E$ be the union of $k-1$ complete graphs on the vertex sets $X_{1}, \ldots, X_{k-1},\left|X_{i}\right|=q r$, respectively. On the other hand let $\mathcal{K}=\mathcal{T}^{+1}(k-1, q r)$. It is easy to see that the pair $E, \mathcal{K}$ is a dominating set in the graph $G_{k, 2}$. Here

$$
|E|=(k-1)\left(\begin{array}{c}
\frac{n}{k-1} \\
2
\end{array}\right)
$$

The total number of pairs not in $E$ is

$$
\left(\begin{array}{c}
k-1 \\
2
\end{array}\right)\left(\frac{n}{k-1}\right)^{2}=\frac{k-2}{2(k-1)} n^{2} .
$$

One member of $\mathcal{K}$ covers exactly $\left(\begin{array}{l}k \\ 2\end{array}\right)-1$ such edges. Hence the size of $\mathcal{K}$ is

$$
\frac{\frac{k-2}{2(k-1)}}{\left(\begin{array}{l}
k \\
2
\end{array}\right)-1} n^{2} .
$$

Adding (19) and (20) the statement of the proposition is obtained.

Proof of Theorem 5. In view of Proposition 1 we only have to give one "small" +1-transversal. Namely a $\mathcal{T}^{+1}(3,5)$ will be given below. Let $X_{1}=\left\{a_{0}, a_{1}, a_{2}, a_{3}, a_{4}\right\}, X_{2}=\left\{b_{0}, b_{1}, b_{2}, b_{3}, b_{4}\right\}, X_{3}=\left\{c_{0}, c_{1}, c_{2}, c_{3}, c_{4}\right\}$. The member $\left\{a_{0}, a_{1}, b_{0}, c_{0}\right\}$ will be illustrated by $(01,0,0)$. Using this notation the members will be

$$
(01,0,0),(3,01,4),(4,0,12)
$$

and all cyclic shifts of these vectors mod 5. By inspection it is easy to see that these 15 subsets form a $\mathcal{T}^{+1}(3,5)$. Actually one can see that the three 4-element sets cover every "distance" mod 5 between any two distinct $X_{i}(1 \leq 3)$, therefore the cyclic shift will cover every pair (between two $X$ 's) exactly once.

Some related work. Balázs Patkós called our attention to the following results which are closely related but use different terminology.

A subset $D_{\mathrm{I}}$ of $V$ is called an independent dominating set in a graph $G=(V, E)$ if it is dominating, and additionally, no two elements of $D_{\mathrm{I}}$ are adjacent. The independent domination number $\gamma_{\mathrm{I}}(G)$ of $G$ is the smallest 
possible size of an independent dominating set. The inequality $\gamma(G) \leq \gamma_{\mathrm{I}}(G)$ is obvious. The independent domination number of $G_{k, \ell}$ was considered in [3]. An independent dominating set here is a family of $k$ and $\ell$-element subsets of $[n]$ where (a) no member is a proper subset of another one (called Sperner family in the related literature) and (b) it is maximal for this property. The authors in [3] exactly determined $\gamma_{\mathrm{I}}\left(G_{3,2}\right)$. Their construction coincides with our construction in the proof of Theorem 4. Denote it by $\mathcal{G} \subset\left(\begin{array}{c}{[n]} \\ 2\end{array}\right) \cup\left(\begin{array}{c}{[n]} \\ 3\end{array}\right)$.

[2] contains a related "stability" result. It is proved that if $\mathcal{F}$ is a nearly optimal family of two- and three-element subsets of $[n]$ that is $\mathcal{F} \subset\left(\begin{array}{c}{[n]} \\ 2\end{array}\right) \cup\left(\begin{array}{c}{[n]} \\ 3\end{array}\right)$ of size $|\mathcal{F}|=\frac{3}{8} n^{2}-o\left(n^{2}\right)$ satisfying (a) and (b) then it must be similar to $\mathcal{G}$ in the following sense:

$$
|\mathcal{F} \triangle \mathcal{G}|=o\left(n^{2}\right)
$$

where $\triangle$ denotes the symmetric difference.

Acknowledgements. We are indebted to Miklós Simonovits for his valuable help. We are also very much obliged to the anonymous referees for the careful reading our paper and correcting the errors.

\section{References}

[1] R.C. Baker, G. Harman, J. Pintz, The difference between consecutive primes. II. Proc. London Math. Soc. (83)(3)(2001) 532562.

[2] D. Gerbner, B. Keszegh, N. Lemons, C. Palmer, D. Pálvölgyi, and B. Patkós, Saturating sperner families. Graphs and Combinatorics, 29(5)(2013)1355-1364.

[3] M. Grüttmüller, S. Hartmann, T. Kalinowski, U. Leck, U. and I.T. Roberts, Maximal Flat Antichains of Minimum Weight, Electronic J. Combinatorics (16)(2009)R69.

[4] H. Hanani, On transversal designs, in: M. Hall Jr., and J.H. Van Lint(Eds.) Combinatorics, pp. 42-52. Mathematical Centre Tracks (55), Amsterdam, 1974.

[5] L. Lovász, M. Simonovits, On the number of complete subgraphs of a graph II, Studies in Pure Mathematics, Springer Basel, 1983, pp. 459495. 
[6] I.Z. Ruzsa, E. Szemerédi, Triple systems with no six points carrying three triangles, in: A. Hajnal, Vera T. Sós(Eds.) Combinatorics (Proc. Fifth Hungarian Colloq. Keszthely, 1976) Vol. II. Colloq. Math. Soc. János Bolyai, (18), North-Holland, Amsterdam, 1978, pp. 939-945.

[7] P. Turán, Egy gráfelméleti szélsőértékfeladatról, Mat. Fiz. Lapok (48)(1941)436-452. 